\title{
Power moments of the error term in the approximate functional equation for $\zeta^{2}(s)$
}

\author{
by
}

\author{
Aleksandar IVić (Beograd)
}

Let as usual $s=\sigma+i t$ be a complex variable, $d(n)$ the number of divisors of $n$, and $\zeta(s)$ the Riemann zeta-function. One may consider (see e.g. Th. 4.2 of [3])

$$
\begin{aligned}
R\left(s ; \frac{t}{2 \pi}\right):=\zeta^{2}(s)-\sum_{n \leq t /(2 \pi)}^{\prime} d(n) n^{-s}-\chi^{2}(s) \sum_{n \leq t /(2 \pi)}^{\prime} d(n) n^{s-1} \\
\quad(0 \leq \sigma \leq 1)
\end{aligned}
$$

as the error term in the approximate functional equation for $\zeta^{2}(s)$, where

$$
\chi(s)=\zeta(s) / \zeta(1-s)=2^{s} \pi^{s-1} \sin (\pi s / 2) \Gamma(1-s) .
$$

In his important works [10], [11] Y. Motohashi established a very precise formula for the function $R(s ; t /(2 \pi))$, which connects it with

$$
\Delta(x):=\sum_{n \leq x}^{\prime} d(n)-x(\log x+2 \gamma-1)-1 / 4,
$$

the error term in the classical divisor problem. Here $\gamma$ is Euler's constant, and in general $\sum_{n \leq y}^{\prime}$ denotes that the last term in the sum is to be halved if $y$ is an integer. In particular, Motohashi has shown that

$$
\chi(1-s) R\left(s ; \frac{t}{2 \pi}\right)=-\sqrt{2}\left(\frac{t}{2 \pi}\right)^{-1 / 2} \Delta\left(\frac{t}{2 \pi}\right)+O\left(t^{-1 / 4}\right) .
$$

By using (1) and the author's bounds (see [2] or Ch. 13 of [3])

$$
\int_{1}^{T}|\Delta(x)|^{A} d x \ll \begin{cases}T^{1+A / 4+\varepsilon}, & 0 \leq A \leq 35 / 4, \\ T^{19 / 54+35 A / 108+\varepsilon}, & A \geq 35 / 4,\end{cases}
$$

1991 Mathematics Subject Classification: 11M06.

Key words and phrases: Riemann zeta-function, approximate functional equation, Voronoï formula for the divisor problem.

Research financed by the Mathematical Institute of Belgrade. 
I. Kiuchi [7] obtained the bounds

$$
\int_{1}^{T}\left|R\left(\frac{1}{2}+i t ; \frac{t}{2 \pi}\right)\right|^{A} d t \ll \begin{cases}T^{1-A / 4+\varepsilon}, & 0 \leq A \leq 4, \\ 1, & A \geq 4\end{cases}
$$

Here, as usual, both $f(x)=O(g(x))$ and $f(x) \ll g(x)$ mean that $|f(x)| \leq$ $C g(x)$ for $x \geq x_{0}, g(x)>0$ and some $C>0$. In the special case $A=2$ a precise result may be obtained. Kiuchi and Matsumoto [8] obtained

$$
\int_{1}^{T}\left|R\left(\frac{1}{2}+i t ; \frac{t}{2 \pi}\right)\right|^{2} d t=\sqrt{2 \pi}\left\{\sum_{n=1}^{\infty} d^{2}(n) h^{2}(n) n^{-1 / 2}\right\} T^{1 / 2}+F(T)
$$

with $F(T)=O\left(T^{1 / 4} \log T\right)$, and I. Kiuchi improved this in [6] to $F(T)=$ $O\left(\log ^{5} T\right)$. In (4) the function $h(n)$ is defined as

$$
h(n)=\left(\frac{2}{\pi}\right)^{1 / 2} \int_{0}^{\infty}(y+n \pi)^{-1 / 2} \cos (y+\pi / 4) d y .
$$

Two integrations by parts give

$$
\begin{aligned}
h(n)=\left(\frac{2}{\pi}\right)^{1 / 2}\left\{-(2 \pi n)^{-1 / 2}\right. & +(2 \pi n)^{-3 / 2} \\
& \left.-\frac{3}{4} \int_{0}^{\infty}(y+n \pi)^{-5 / 2} \cos (y+\pi / 4) d y\right\},
\end{aligned}
$$

which easily yields

(6) $h(n)=-\frac{n^{-1 / 2}}{\pi}+\frac{n^{-3 / 2}}{2 \pi^{2}}+O\left(n^{-5 / 2}\right), \quad h(n)<0 \quad(n \in \mathbb{N})$,

so that the series in (4) converges, since $d(n) \ll n^{\varepsilon}$ for any $\varepsilon>0$.

The object of this note is to improve (3), and at the same time to indicate how a simple proof of (4) with $F(T)=O\left(\log ^{5} T\right)$ may be obtained. The results are contained in the following

Theorem. Let $A \geq 0$ be a given constant. For $0 \leq A<4$ there exists a positive constant $C(A)$ such that

$$
\int_{1}^{T}\left|R\left(\frac{1}{2}+i t ; \frac{t}{2 \pi}\right)\right|^{A} d t \sim C(A) T^{1-A / 4} \quad(T \rightarrow \infty) .
$$

Moreover, there exist effectively computable constants $B>0$ and $D$ such that, for any $\varepsilon>0$,

$$
\int_{1}^{T}\left|R\left(\frac{1}{2}+i t ; \frac{t}{2 \pi}\right)\right|^{4} d t=B \log T+D+O\left(T^{\varepsilon-1 / 23}\right),
$$


and for $A>4$

$$
\int_{1}^{T}\left|R\left(\frac{1}{2}+i t ; \frac{t}{2 \pi}\right)\right|^{A} d t=D(A)+O(E(T, A))
$$

where

$$
D(A)=\int_{1}^{\infty}\left|R\left(\frac{1}{2}+i t ; \frac{t}{2 \pi}\right)\right|^{A} d t
$$

is finite and positive, and

$$
E(T, A)= \begin{cases}T^{1-A / 4}, & 4<A<28 / 3 \\ T^{(4-2 A) / 11+\varepsilon}, & A \geq 28 / 3\end{cases}
$$

Proof. We begin with the case $A>4$, which is not difficult to settle. Instead of (2) we may use the bounds

$$
\int_{1}^{T}|\Delta(x)|^{A} d x \ll \begin{cases}T^{1+A / 4+\varepsilon}, & 0 \leq A \leq 28 / 3, \\ T^{1+7(A-2) / 22+\varepsilon}, & A \geq 28 / 3 .\end{cases}
$$

This result is obtained in the same way as (2) was obtained, only instead of $\Delta(x) \ll x^{35 / 108+\varepsilon}$ one uses the sharper estimate $\Delta(x) \ll x^{7 / 22+\varepsilon}$ of Iwaniec and Mozzochi [5], e.g. in (13.71) of [3] and in the estimate that follows it. Moreover, from the proof of D. R. Heath-Brown [1] one obtains then

$$
\int_{1}^{T}|\Delta(x)|^{A} d x \ll \begin{cases}T^{1+A / 4}, & 0 \leq A<28 / 3, \\ T^{1+7(A-2) / 22+\varepsilon}, & A \geq 28 / 3,\end{cases}
$$

and in the bound for $A \geq 28 / 3$ one could actually replace $T^{\varepsilon}$ by a suitable power of the logarithm. Since $|\chi(1 / 2 \pm i t)|=1$, it follows from (1) and (12) that

$$
\int_{Y}^{2 Y}\left|R\left(\frac{1}{2}+i t ; \frac{t}{2 \pi}\right)\right|^{A} d t \ll Y^{1-A / 4}+Y^{-A / 2} \int_{Y}^{2 Y}|\Delta(y)|^{A} d y \ll E(Y, A) .
$$

This easily yields (9), since both exponents of $T$ in the definition (11) of $E(T, A)$ are negative for $A>4$.

To obtain the remaining results of the Theorem it is necessary to use the classical Voronoï formula for $\Delta(x)$ (see Ch. 3 of [3]), namely

$$
\Delta(x)=(\pi \sqrt{2})^{-1} x^{1 / 4} \sum_{n=1}^{\infty} d(n) n^{-3 / 4} \cos (4 \pi \sqrt{x n}-\pi / 4)+O\left(x^{-1 / 4}\right),
$$

which in truncated form may be written as

$$
\begin{aligned}
\Delta(x)=(\pi \sqrt{2})^{-1} x^{1 / 4} \sum_{n \leq N} d(n) n^{-3 / 4} \cos ( & 4 \pi \sqrt{x n}-\pi / 4) \\
& +O\left(x^{\varepsilon}+x^{1 / 2+\varepsilon} N^{-1 / 2}\right)
\end{aligned}
$$


for any given $\varepsilon>0$, and $1 \leq N \leq x^{C}$, where $C>0$ is any fixed number. The key idea, suggested by (1), is to make the connection between the functions $R(\cdot)$ and $\Delta(\cdot)$ in such a way that the appropriate analogues of (13) and (14) may by obtained for $R(\cdot)$. The relation (1) is too weak for this purpose, and we shall use the following formula which follows from Motohashi's work (e.g. pp. $74-75$ of [11]):

$$
\begin{aligned}
\chi\left(\frac{1}{2}-i t\right) R\left(\frac{1}{2}+i t ; \frac{t}{2 \pi}\right) \\
=-\sqrt{2}\left(\frac{t}{2 \pi}\right)^{-1 / 2} \Delta\left(\frac{t}{2 \pi}\right) \\
+(\pi \sqrt{2})^{-1}\left(\frac{t}{2 \pi}\right)^{-1 / 2}\left(\frac{1}{6} \log \left(\frac{t}{2 \pi}\right)+\frac{\gamma}{3}+1\right) \\
+(2 \pi)^{-1 / 2}\left(\frac{t}{2 \pi}\right)^{-1 / 4} \sum_{n=1}^{\infty} d(n) n^{-1 / 4} h_{1}(n) \cos (2 \sqrt{2 \pi t n}-\pi / 4) \\
+O\left(t^{-3 / 4}\right),
\end{aligned}
$$

where

$$
h_{1}(n):=\int_{0}^{\infty}(y+n \pi)^{-3 / 2} \cos (y-\pi / 4) d y \ll n^{-3 / 2} .
$$

Now we define

$$
g(t):=t^{1 / 2} \chi\left(\frac{1}{2}-i t\right) R\left(\frac{1}{2}+i t ; \frac{t}{2 \pi}\right),
$$

so that $g(t)$ is real for $t$ real, and

$$
|g(t)|=t^{1 / 2}\left|R\left(\frac{1}{2}+i t ; \frac{t}{2 \pi}\right)\right| .
$$

Noting that an integration by parts gives

$$
h_{1}(n)=\left(\frac{2}{\pi n}\right)^{1 / 2}+(2 \pi)^{1 / 2} h(n),
$$

where $h(n)$ is given by (5), we deduce from (13) and (15) that

$$
\begin{aligned}
g(t) & -(6 \sqrt{\pi})^{-1}\left(\log \frac{t}{2 \pi}+2 \gamma+6\right) \\
& =(2 \pi t)^{1 / 4} \sum_{n=1}^{\infty} d(n) h(n) n^{-1 / 4} \cos (2 \sqrt{2 \pi t n}-\pi / 4)+O\left(t^{-1 / 4}\right) .
\end{aligned}
$$


On the other hand, by using (14) and the fact that

$$
\sum_{n>N} d(n) n^{-1 / 4} h_{1}(n) \cos (2 \sqrt{2 \pi t n}-\pi / 4) \ll N^{-3 / 4} \log N,
$$

we obtain from (15), for $1 \leq N \leq t^{C}$,

$$
\begin{gathered}
g(t)-(6 \sqrt{\pi})^{-1}\left(\log \frac{t}{2 \pi}+2 \gamma+6\right) \\
=(2 \pi t)^{1 / 4} \sum_{n \leq N} d(n) h(n) n^{-1 / 4} \cos (2 \sqrt{2 \pi t n}-\pi / 4) \\
\quad+O\left(t^{\varepsilon}+t^{1 / 2+\varepsilon} N^{-1 / 2}\right) .
\end{gathered}
$$

If we now set

$$
G(t):=g(t)-(6 \sqrt{\pi})^{-1}\left(\log \frac{t}{2 \pi}+2 \gamma+6\right),
$$

then the analogy between $\Delta(x)$ and $G(t)$ is indeed striking: (13) corresponds to (18) and (14) to (19), only the scaling factors are different and $n^{-3 / 4}$ is replaced by

$$
n^{-1 / 4} h(n) \sim-\pi^{-1} n^{-3 / 4} .
$$

Thus essentially the results on $\Delta(x)$ based only on the use of (13) and (14) have their counterparts for $G(t)$, and through the use of (16) and (20) one can then obtain the corresponding results for $R(1 / 2+i t ; t /(2 \pi))$. To stress our point, note that the result

$$
\Delta(x)-\Delta(y) \ll(x+y)^{\varepsilon}(|x-y|+1) \quad(x, y \geq 1),
$$

which follows trivially from $d(n) \ll n^{\varepsilon}$ and the definition of $\Delta(x)$, does not seem obtainable by (13) or (14). Thus we cannot infer automatically the corresponding bound

$$
G(x)-G(y) \ll(x+y)^{\varepsilon}(|x-y|+1) \quad(x, y \geq 1)
$$

for $G(t)$ (or $g(t)$ ). Indeed, it is not obvious how the last bound can be proved.

After the above discussion it is easy to see why (4) holds with $F(T)=$ $O\left(\log ^{5} T\right)$. Namely T. Meurman [9] proved

$$
\int_{1}^{X} \Delta^{2}(x) d x=\frac{\zeta^{4}(3 / 2)}{6 \pi^{2} \zeta(3)} X^{3 / 2}+R(X)
$$

with $R(X)=O\left(X \log ^{5} X\right)$. This was obtained much earlier by K.-C. Tong [13], but Meurman's method is substantially simpler than Tong's. E. Preissmann [12] indicated how at one place in the proof a variant of Hilbert's inequality may be used to save a further log-power, so that now even $R(X)=$ $O\left(X \log ^{4} X\right)$ is known. Since the works of Meurman and Preissmann use 
only (13) and (14), it follows that the analogue of (21) may be obtained for $G(t)$, and this will be

$$
\int_{1}^{T} G^{2}(t) d t=A_{1} T^{3 / 2}+R_{1}(T), \quad R_{1}(T)=O\left(T \log ^{4} T\right),
$$

with the value

$$
A_{1}=\frac{\sqrt{2 \pi}}{3} \sum_{n=1}^{\infty} d^{2}(n) h^{2}(n) n^{-1 / 2} .
$$

From (20) and (22) one obtains

$$
\begin{aligned}
\int_{1}^{T} g^{2}(t) d t= & \int_{1}^{T} G^{2}(t) d t+(3 \sqrt{\pi})^{-1} \int_{1}^{T} G(t)\left(\log \frac{t}{2 \pi}+2 \gamma+6\right) d t \\
& +(36 \pi)^{-1} \int_{1}^{T}\left(\log \frac{t}{2 \pi}+2 \gamma+6\right)^{2} d t \\
= & A_{1} T^{3 / 2}+R_{2}(T),
\end{aligned}
$$

say, where

(23) $R_{2}(T)=R_{1}(T)+O\left(T^{3 / 4} \log T\right)+(36 \pi)^{-1} \int_{1}^{T}\left(\log \frac{t}{2 \pi}+2 \gamma+6\right)^{2} d t$,

since by the first derivative test (Lemma 2.1 of [3]) one easily finds that

$$
H(T):=\int_{1}^{T} G(t)\left(\log \frac{t}{2 \pi}+2 \gamma+6\right) d t \ll T^{3 / 4} \log T .
$$

Thus we have $R_{2}(T)=O\left(T \log ^{4} T\right)$, so that (17) and integration by parts give

$$
\int_{1}^{T}\left|R\left(\frac{1}{2}+i t ; \frac{t}{2 \pi}\right)\right|^{2} d t=3 A_{1} T^{1 / 2}+F(T)
$$

with

$$
F(T)=R_{2}(T) T^{-1}-R_{2}(1)-3 A_{1}+\int_{1}^{T} R_{2}(t) t^{-2} d t .
$$

Hence the bound $R_{2}(T)=O\left(T \log ^{4} T\right)$ gives immediately (4) with $F(T)=$ $O\left(\log ^{5} T\right)$, obtained by I. Kiuchi [6], whose proof is much more involved, as it reproduces the details of the method of Meurman and Preissmann. Note also that if

$$
R_{1}(T)=o\left(T \log ^{2} T\right) \quad(T \rightarrow \infty)
$$


could be proved, then from (23) and (25) it would follow that

$$
F(T)=\left(\frac{1}{108 \pi}+o(1)\right) \log ^{3} T \quad(T \rightarrow \infty) .
$$

This would mean the appearance of a new main term in (4), and a similar observation was made by Kiuchi [6]. It may also be remarked that by the method of [4] it follows that there exist constants $B_{1}, B_{2}>0$ such that for $T \geq T_{0}$ every interval $\left[T, T+B_{1} T^{1 / 2}\right]$ contains points $t_{1}, t_{2}$ such that

$$
H\left(t_{1}\right)>B_{2} t_{1}^{3 / 4} \log t_{1}, \quad H\left(t_{2}\right)<-B_{2} t_{2}^{3 / 4} \log t_{2},
$$

where $H(t)$ is defined by (24), and a sharp mean square formula for $H(t)$ may be also derived. This observation coupled with the bound in (24) prompts one to state the optimistic conjecture that

$$
\begin{aligned}
& \int_{1}^{T}\left|R\left(\frac{1}{2}+i t ; \frac{t}{2 \pi}\right)\right|^{2} d t \\
& \quad=3 A_{1} T^{1 / 2}+a_{0} \log ^{3} T+a_{1} \log ^{2} T+a_{2} \log T+a_{3}+O\left(T^{\varepsilon-1 / 4}\right)
\end{aligned}
$$

holds with any $\varepsilon>0$, and effectively computable constants $a_{0}, a_{1}, a_{2}$ and $a_{3}$.

We return now to the proof of the Theorem. K.-M. Tsang [14] recently proved

$$
\int_{1}^{X} \Delta^{4}(x) d x=3 c_{2}\left(64 \pi^{4}\right)^{-1} X^{2}+O\left(X^{45 / 23+\varepsilon}\right)
$$

with

$$
c_{2}=\sum_{\substack{k, l, m, n=1 \\ k^{1 / 2}+l^{1 / 2}=m^{1 / 2}+n^{1 / 2}}}^{\infty}(k l m n)^{-3 / 4} d(k) d(l) d(m) d(n),
$$

which he showed to be a convergent series. Tsang's proof is entirely based on (14), hence we may follow it to derive the corresponding result for $g(t)$, which will be

$$
\int_{1}^{T} g^{4}(t) d t=\frac{3 \pi}{8} c_{3} T^{2}+U(T), \quad U(T)=O\left(T^{45 / 23+\varepsilon}\right),
$$

where

$$
c_{3}:=\sum_{\substack{k, l, m, n=1 \\ k^{1 / 2}+l^{1 / 2}=m^{1 / 2}+n^{1 / 2}}}^{\infty}(k l m n)^{-1 / 4} h(k) h(l) h(m) h(n) d(k) d(l) d(m) d(n) .
$$


Since $h(n)<0$ and $h(n) \ll n^{-1 / 2}$, one shows that $c_{3}$ is finite and positive in the same way as Tsang did for $c_{2}$ in (28). Using (17) and integrating (29) by parts we easily obtain (8) with

$$
B=\frac{3 \pi c_{3}}{4}>0, \quad D=2 \int_{1}^{\infty} \frac{U(t)}{t^{3}} d t-U(1) .
$$

Let now $0 \leq A<4$. From (4), (29) and Hölder's inequality for integrals it follows that

$$
T^{1-A / 4} \ll \int_{1}^{T}\left|R\left(\frac{1}{2}+i t ; \frac{t}{2 \pi}\right)\right|^{A} d t \ll T^{1-A / 4} \quad(0 \leq A<4) .
$$

D. R. Heath-Brown [1] proved the existence of

$$
\lim _{X \rightarrow \infty} X^{-1-k / 4} \int_{1}^{X}|\Delta(x)|^{k} d x
$$

for $0 \leq k \leq 9$ by a general method. In view of (17) and (31) this method gives, when applied to $g(t)$, the existence of

$$
\lim _{T \rightarrow \infty} T^{-1-k / 4} \int_{1}^{T}|g(t)|^{k} d t
$$

for $0 \leq k<4$. From (17) and integration by parts we deduce that

$$
C(A)=\lim _{T \rightarrow \infty} T^{A / 4-1} \int_{1}^{T}\left|R\left(\frac{1}{2}+i t ; \frac{t}{2 \pi}\right)\right|^{A} d t
$$

exists for $0 \leq A<4$. Since (31) holds we obtain $C(A)>0$, hence (7) is proved.

\section{References}

[1] D. R. Heath-Brown, The distribution and moments of the error term in the Dirichlet divisor problem, Acta Arith. 60 (1992), 389-415.

[2] A. Ivić, Large values of the error term in the divisor problem, Invent. Math. 71 (1983), 513-520.

[3] —, The Riemann Zeta-function, Wiley, New York, 1985.

[4] —, Large values of certain number-theoretic error terms, Acta Arith. 56 (1990), $135-159$.

[5] H. Iwaniec and C. J. Mozzochi, On the divisor and circle problems, J. Number Theory 29 (1988), 60-93.

[6] I. Kiuchi, An improvement on the mean value formula for the approximate functional equation of the square of the Riemann zeta-function, ibid., to appear.

[7] - Power moments of the error term for the approximate functional equation of the Riemann zeta-function, Publ. Inst. Math. (Beograd) 52 (66) (1992), in print. 
[8] I. Kiuchi and K. Matsumoto, Mean value results for the approximate functional equation of the square of the Riemann zeta-function, Acta Arith. 61 (1992), 337-345.

[9] T. Meurman, On the mean square of the Riemann zeta-function, Quart. J. Math. Oxford Ser. (2) 38 (1987), 337-343.

[10] Y. Motohashi, A note on the approximate functional equation for $\zeta^{2}(s)$, Proc. Japan Acad. Ser. A 59 (1983), 393-396 and II, ibid. 469-472.

[11] - Lectures on the Riemann-Siegel Formula, Ulam Seminar, Dept. Math., Colorado University, Boulder, 1987.

[12] E. Preissmann, Sur la moyenne quadratique du terme de reste du problème du cercle, C. R. Acad. Sci. Paris 306 (1988), 151-154.

[13] K.-C. Tong, On divisor problem III, Acta Math. Sinica 6 (1956), 515-541 (in Chinese).

[14] K.-M. Tsang, Higher power moments of $\Delta(x), E(t)$ and $P(x)$, Proc. London Math. Soc. (3) 65 (1992), 65-84.

KATEDRA MATEMATIKE RGF-A

UNIVERSITETA U BEOGRADU

DJUŠINA 7

11000 BEOGRAD, SERBIA (YUGOSLAVIA) 